\title{
Suckling Piglet Intestinal Enterocyte Nutrient Metabolism Changes
}

\author{
Qiye Wang ${ }^{\mathrm{a}}$ Xia Xiong ${ }^{\mathrm{b}}$ Xiaocheng Wang ${ }^{\mathrm{b}}$ Qiang Tu ${ }^{\mathrm{a}, \mathrm{c}}$ Jianzhong $\mathrm{Li}^{\mathrm{a}}$ \\ Xueqin Ding ${ }^{\mathrm{a}}$ Yali Li ${ }^{\mathrm{a}}$ Pengfei Huang ${ }^{\mathrm{a}}$ Shanping He ${ }^{\mathrm{a}}$ Yulong Yin ${ }^{\mathrm{a}, \mathrm{b}}$ \\ Huansheng Yang ${ }^{\mathrm{a}, \mathrm{b}}$
}

aHunan International Joint Laboratory of Animal Intestinal Ecology and Health, Animal Nutrition and Human Health Laboratory, School of Life Sciences, Hunan Normal University, Changsha, ${ }^{b} K e y$ Laboratory of Agro-Ecological Processes in Subtropical Region, Hunan Provincial Engineering Research Center of Healthy Livestock, Scientific Observing and Experimental Station of Animal Nutrition and Feed Science in South-Central, Ministry of Agriculture, Institute of Subtropical Agriculture, Chinese Academy of Sciences, Changsha, 'Shandong University-Helmholtz Institute of Biotechnology, State Key Laboratory of Microbial Technology, School of Life Science, Shandong University, Jinan, China

\section{Key Words}

Differentiated enterocytes $•$ Intestine $・$ Nutrient metabolism $•$ Piglet $•$ Suckling period

\begin{abstract}
Background/Aims: Intestinal morphology and the types of enterocytes are changed in piglets during the suckling period, but it is unclear whether these changes are associated with metabolic changes in epithelium. The present study was conducted to test the hypothesis that glucose, fatty acids, and amino acid metabolism in differentiated piglet enterocytes changed during suckling. Methods: Twenty-four piglets (Duroc $\times$ [Landrace $\times$ Yorkshire]) from 8 litters (3 piglets/litter) were selected. A single piglet from each litter was randomly selected and euthanized at days 7, 14, and 21. Differentiated enterocytes (DE) were isolated from their mid-jejunum. Isobaric tags for relative and absolute quantification and subsequent liquid chromatography-tandem mass spectrometry were used to identify and measure protein synthesis. Results: The results showed that various activities, including: cellular processes; metabolic processes; biological regulation; pigmentation; and, localization, in DEs changed during suckling. Metabolic process analyses revealed that protein expression related to glycolysis and citrate cycle was decreased from day 7 to day 14 . The number of differentiated enterocytes of $21 \mathrm{~d}$ piglets increased compared to $7 \mathrm{~d}$ piglets. Most of the proteins involved in fatty acid and amino acids metabolism had decreased DE expression between day 7 and day 14. Some, but not all, detected proteins down-regulated in DEs of 21 day piglets compared to 7 day piglets. Conclusion: These results indicate that glucose, fatty acids, and amino acids metabolism changed during suckling. This may provide useful information for designing feed formulas and regulating piglet intestinal growth and development.




\section{Introduction}

Piglet gastrointestinal systems develop intensively during their first few weeks $[1,2]$. The most intensive changes occur in the intestinal epithelium [2,3]. Villi, and crypt, depth increase rapidly post-partum. Villi shape changes during the early postnatal period $[1,4,5]$. Intestinal enterocytes, which constitute more than $90 \%$ of all intestinal epithelial cells, play key roles in digesting and absorbing luminal nutrients. Vacuolated fetal-type enterocytes are replaced by adult-type enterocytes in piglets during suckling [6]. Studies to date that were conducted to investigate piglet intestinal development have focused on intestinal growth, morphology, and functional changes [3-6]. It remains unclear whether intestinal morphology or enterocyte type changes in suckling piglets associate with epithelial metabolic change.

The intestine has a high rate of nutrient utilization via the portal-drained viscera (PDV) which includes the stomach, intestines, pancreas, and spleen. It contributes about $5 \%$ of body weight and accounts for $20-35 \%$ of whole-body protein synthesis and energy expenditure. Most nutrients are utilized by the intestinal epithelium [7-9]. About one-third of all essential dietary amino acids are consumed by the intestine during first-pass metabolism. It has been reported that small intestine growth and development depends largely on luminal nutrient composition [3, 4]. Understanding nutrient metabolism in the intestinal epithelium is central to designing milk replacement formulas and regulating gastrointestinal system growth and development [3].

Using a label-free quantitative proteomics approach, Hansson et al. found that carbohydrate, amino acids, lipid, fatty acids, and steriod metabolisms changed in mice intestinal epithelial cells during suckling [10]. There are marked differences between early life rodent and piglet intestinal maturation stages [11]. The intestinal epithelium undergoes continual renewal along the crypt-villus axis. Nutrient metabolism differs between villus and crypt epithelial cells in piglets $[2,12,13]$. Differentiated enterocytes, located in the upper villi, are the primary intestinal epithelial cells involved in nutrient digestion and absorption $[2,14]$. This experiment is premised on thehypothesis that glucose, fatty acids, and amino acids metabolism in DEs in piglets change during suckling. The current study purposed to investigate nutrient metabolism in sucking piglet upper villus enterocytes.

\section{Materials and Methods}

The experimental design and procedures in this study were reviewed and approved by the Animal Care and Use Committee of the Institute of Subtropical Agriculture, Chinese Academy of Science.

Reagents

DL- $\beta$-Hydroxybutyrate sodium salt was purchased from J\&K Chemical (Ltd., USA). Trypsin was procured from Promega (Madison, WI, USA). iTRAQ-reagent was purchased from Applied Biosystems (Foster City, CA, USA). Bovine serum alumin (BSA, fraction V), phenylmethylsulfonyl fluoride (PMSF), dithiothreitol (DTT), and other chemical were obtained from Sigma-Aldrich (St. Louis, MO, USA) unless otherwise stated.

\section{Animals and intestinal upper villus enterocytes isolation}

Twenty-four piglets (Duroc $\times$ [Landrace $\times$ Yorkshire]) from 8 litters ( 3 piglets per litter) were selected based on their BW and gender. Sows had free access to feed and drinking water. Piglets had free access to nipples and water during the experiment. One piglet from each litter was maintained under general anesthesia and intravenously euthanized via a jugular injection of $4 \%$ sodium pentobarbital solution (40 $\mathrm{mg} / \mathrm{kg}$ body weight) at 7, 14, and 21 days of age. The DEs were isolated from jejunum upper villi using distended intestinal sac method as previously described $[15,16]$. Ice-cold physiological saline solution rinsed jejunum segments are incubated with oxygenated phosphate buffered saline at $37{ }^{\circ} \mathrm{C}$ for 30 min. Intestine segments were incubated in an oxygenated isolation buffer (5 mM Na2EDTA, $10 \mathrm{mM}$ HEPES pH 
7.4, $0.5 \mathrm{mM}$ DTT, 0.25\% BSA, $2.5 \mathrm{mM}$ D-glucose, $2.5 \mathrm{mM}$ L-glutamine, $0.5 \mathrm{mM}$ dl- $\beta$-hydroxybutyrate sodium salt, oxygenated with an $\mathrm{O}_{2} / \mathrm{CO}_{2}$ mixture $(19: 1, \mathrm{v} / \mathrm{v})$ ) at $37^{\circ} \mathrm{C}$ for $40 \mathrm{~min}$ to isolate upper villus enterocytes. The isolation buffers were collected and centrifuged at $400 \mathrm{~g}$ for $4 \mathrm{~min}$ at $4{ }^{\circ} \mathrm{C}$. The collected enterocytes were twice washed with an oxygenated cell suspension buffer (10 mM HEPES, $1.5 \mathrm{mM} \mathrm{CaCl}, 2.0 \mathrm{mM} \mathrm{MgCl}_{2}$, $\mathrm{pH}$ 7.4). The isolated upper villus enterocytes were confirmed by testing alkaline phosphatase activity and proliferating cell nuclear antigen (PCNA) expression.

\section{Sample preparation and isobaric labeling}

A lysis buffer (7 M urea, 2 M thiourea, 4\% w/v 3-[(3-Cholamidopropyl) dimethylammonio] propanesulfonate, 20 mMTributyl phosphate, and 0.2\% Bio-lyte ( $\mathrm{pH} 3-10)$ ) and a protease inhibitor cocktail (Roche Diagnostics Ltd, Mannheim, Germany) re-suspended and disrupted the isolated enterocytes. The lysate was then treated with DNAse I and RNAse A at final concentrations of $1 \mathrm{mg} / \mathrm{mL}$ and $0.25 \mathrm{mg} / \mathrm{mL}$, respectively. The protein solution was centrifugally separated from the cell debris at $12,000 \times \mathrm{g}, 4{ }^{\circ} \mathrm{C}$ for 5 min. A Ready Prep 2-D Cleanup Kit (Bio-Rad Laboratories, USA) was used to further purify the crude protein. Purified protein underwent a reductive alkylation reaction. The protein concentration was measured using a 2-D Quant Kit (GE Healthcare, USA). Trypsin digestion and iTRAQ labeling were performed according to manufacturer protocol (Applied Biosystems, Foster City, CA, USA). A sample of $100 \mu \mathrm{g}$ total protein from each was reduced, alkylated, and digested overnight with trypsin (Promega, Madison, WI, USA) at $37{ }^{\circ} \mathrm{C}$. It was then labeled with iTRAQ-reagents (Applied Biosystems, Foster City, CA, USA) as follows: 7d, iTRAQ reagent $115 ; 14 \mathrm{~d}$, iTRAQ reagent 116; 21d, iTRAQ reagent 117.

\section{Peptide fractionation and LC-MS/MS acquisition}

The isotopically-labeled samples were pooled. An Ultremex SCX column, containing 5- $\mu$ m particles (Phenomenex, USA), fractionated the pooled samples into 12 fractions. A Strata X C18 column (Phenomenex, USA) desalted the eluted fractions. The desalted fractions were then vacuum-dried. Average peptide concentration in each fraction was adjusted to $0.25 \mu \mathrm{g} / \mu \mathrm{L}$. Dried peptides were stored at $-80{ }^{\circ} \mathrm{C}$ until used for MS analysis. The analytical separation was performed using a nanospray ion source (Waters, USA) system (coupled with Triple TOF). Symmetry C18- packed microfluidic traps (5 $\mu \mathrm{m}, 180 \mu \mathrm{m} \times 20 \mathrm{~mm})$ and nanofluidic columns were employed in online trapping, desalting. BEH130 C18-packed (1.7 $\mu \mathrm{m}, 100 \mu \mathrm{m}$ $\times 100 \mathrm{~mm}$ ) nanofluidic columns were used for analytical separation. Water/acetonitrile/formic acid (A: 98/2/0.1\%; B: 2/98/0.1\%) composed solvents were used. A portion of the $2.25 \mu \mathrm{g}(9 \mu \mathrm{L})$ sample was loaded, trapped, and desalted. Analytical separation was established by maintaining $5 \%$ B for $1 \mathrm{~min}$ at a $300 \mathrm{~nL} / \mathrm{min}$ flow rate. The following then occured: a linear gradient to $35 \%$ B occurred at $40 \mathrm{~min}$, this gradient increased to $80 \%$ B at $45 \mathrm{mi}$; and was maintained for 5 min after the peptide elution window. Initial chromatographic conditions were restored in $2 \mathrm{~min}$.

A Triple TOF 5600 System (AB SCIEX, USA), fitted with a Nanospray III source (AB SCIEX, USA) and a pulled quartz tip as the emitter (New Objectives, USA), was used for data acquisition. Data was acquired under the condition of an ion spray voltage of $2.5 \mathrm{kV}$, curtain gas of $30 \mathrm{Psi}$, nebulizer gas of 15 Psi, and an interface heater temperature of $150{ }^{\circ} \mathrm{C}$. The MS was operated with a RP $\geq 30,000$ FWHM for the TOF MS scans. Information dependant acquisition (IDA) survey scans were acquired in $250 \mathrm{~ms}$ and as many as 30 product ion scans were collected if they exceeded a 120 counts per second (counts/s) threshold with a $2+$ to $5+$ charge-state. Total cycle time was fixed to $3.3 \mathrm{~s}$ and the Q2 transmission window was 100 Da for $100 \%$.

Four time bins were summed for each scan at a pulser frequency value of $11 \mathrm{kHz}$ via monitoring of the $40 \mathrm{GHz}$ multichannel TDC detector with four-anode/channel detection. A sweeping collision energy setting of $35 \pm 5 \mathrm{eV}$ coupled with iTRAQ adjusted rolling collision energy was applied to all precursor ions for collision-induced dissociation. Dynamic exclusion was set for $1 / 2$ of the peak width (18 s). The precursor was then refreshed off the exclusion list.

\section{Database analysis and quantification}

Mascot software (version 2.3.02, Matrix Science) was used to identify and quantify proteins. Searches were made against the NCBI non-redundant database consisting of Sus scrofa proteins. Spectra from the 12 fractions of each sample were combined into one MGF (Mascot generic format) file after the raw data was loaded. The MGF file was searched using the following search parameters: i) trypsin was selected as the enzyme with one missed cleavage allowed; ii) the fixed modifications of carbamidomethylation were set as 
Cys; iii) peptide tolerance was set as $0.05 \mathrm{Da}$; and, iv) MS/MS tolerance set at $0.1 \mathrm{Da}$. An automatic decoy database search strategy was used to estimate the false discovery rate (FDR). False positive matches divided by the total matches were employed to calculate FDR. The final FDR was less than $1.5 \%$. Search results were passed through additional filters prior to data exportation. The filters used for protein identification were: 1) significance threshold $P<0.05$ (with 95\% confidence); and, 2) ion score or expected cutoff $<$ 0.05 (with 95\% confidence). The filters for protein quantitation were: 1) "median" was used for the protein ratio type; 2 ) the minimum precursor charge was set as $2+$ and the minimum peptides were set as 2 . Only 2 or more than 2 unique peptides were used to quantify proteins using IQuant (BGI-Shenzhen, China). The median intensities were set as normalization, and outliers were removed automatically. In the present study, a protein with more than 1.2 -fold or less than 0.8 -fold difference between day 7 and day 14 , or day 21 , and a $P$-value $\leq 0.05$ (using t-test) was deemed differentially expressed (IQuant, China).

\section{Bioinformatics analysis}

The Blast2GO program was used for functional annotations of the differentially expressed proteins against non-redundant database consisting of Sus scrofa proteins [17]. The differentially-expressed protein biological process ontology was performed using WEGO program [18]. The metabolic pathway analysis was performed using the Kyoto Encyclopedia of Genes and Genomes (KEGG) Pathway database.

\section{Results}

Gene ontology category
and KEGG pathway
analysis
We identified 2417 unique
proteins. Of these, 1269 were differentially synthesized between day 7 and day 14; or day 7 and day 21. Differentiallyexpressed protein functional annotations were classified in terms of biological process with a WEGO program for the overrepresentation of specific gene ontology (GO) terms. Differentiallyexpressed proteins were primarily involved in cellular process, metabolic process, biological regulation, pigmentation, localization, multicellular organismal process, cellular component organization, establishment of localization, response to stimulus, developmental process (Fig. 1). A KEGG pathway enrichment analysis showed that the differentially expressed proteins were primarily involved in ribosome, carbon metabolism, protein processing in endoplasmic reticulum,

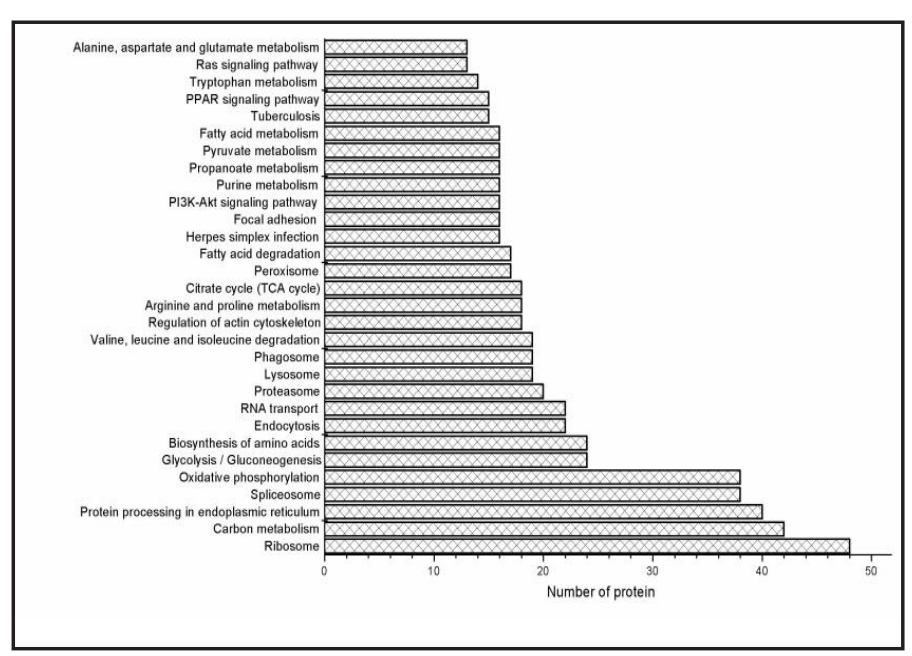

Fig. 1. Functional categories of differentially-expressed proteins in suckling pig jejunal upper villus enterocytes. Gene ontology (GO) biological processes for significantly differentially-expressed proteins performed using WEGO.

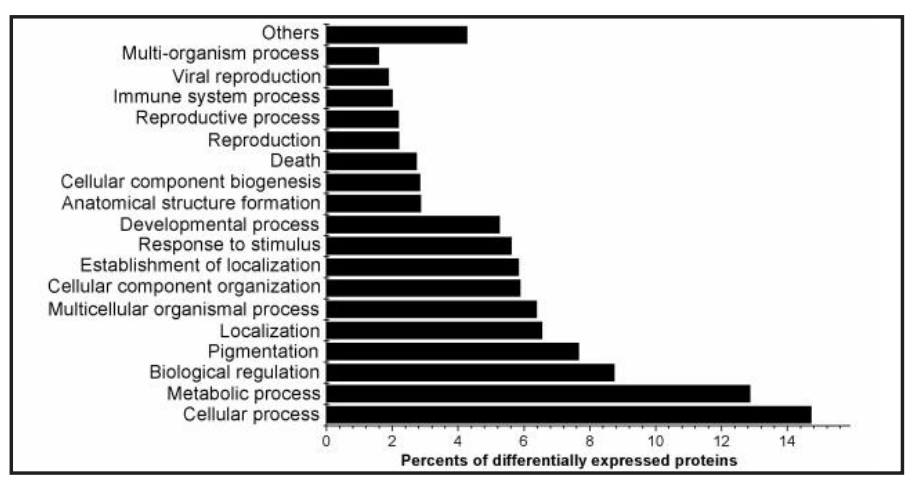

Fig. 2. Top Kyoto Encyclopedia of Genes and Genomes pathways enriched with differentially-expressed suckling piglet jejunal upper villus enterocyte proteins. 
Table 1. Abundance changes of proteins involved in glycolysis injejunal upper villus enterocytes of suckling piglets

\begin{tabular}{|c|c|c|c|}
\hline \multirow[b]{2}{*}{ Proteins } & \multirow[b]{2}{*}{$\begin{array}{c}\text { KEGG } \\
\text { Orthology }\end{array}$} & \multicolumn{2}{|c|}{ Fold change } \\
\hline & & $\begin{array}{c}\text { D14 vs } \\
\text { D7 }\end{array}$ & $\begin{array}{c}\text { D21 vs } \\
\text { D7 }\end{array}$ \\
\hline Akcohol dehydrogenase $\left(\mathrm{NADP}^{+}\right)$ & K00002 & 0.85 & 1.56 \\
\hline L-kactate dehydrogenase & к00016 & 1.25 & 1.43 \\
\hline $\begin{array}{l}\text { S-(hydroxymethyl)glutathione dehydrogenase / Akohol } \\
\text { dehydrogenase }\end{array}$ & К00121 & 0.72 & 1.54 \\
\hline Adehyde de hydrogenase $\left(\mathrm{NAD}^{+}\right)$ & ко0128 & 0.58 & 0.93 \\
\hline Glyceraldehyde 3-phosphate dehydrogenase & ко0134 & 0.74 & 1.31 \\
\hline Addehyde dehydrogenase family 9 member A1 & К00149 & 0.94 & 1.28 \\
\hline Pyruvate dehydrogenase E1 component subunit alpha & ко0161 & 1.68 & 2.25 \\
\hline Pyruvate dehydrogenase E1 component subunit beta & к00162 & 0.57 & 1.26 \\
\hline Hexokinase & ко0844 & 1.75 & 1.65 \\
\hline 6-phosphofructokinase 1 & ко0850 & 1.45 & 1.43 \\
\hline Pyruvate kinase & к00873 & 1.55 & 2.26 \\
\hline Phosphoenolpyruvate carboxykinase (GTP) & к01596 & 0.70 & 0.77 \\
\hline Fructose-bisphosphate aldohse, class I & к01623 & 1.62 & 1.67 \\
\hline Enolase & к01689 & 0.97 & 1.22 \\
\hline Aldose 1-epimerase & к01785 & 0.70 & 1.36 \\
\hline Triosephosphate isomerase (TIM) & к01803 & 0.93 & 1.33 \\
\hline Glucose-6-phosphate isomerase & ко1810 & 1.19 & 1.26 \\
\hline 2,3-bisphosphoglycerate-dependent phosphoglycerate mutase & ко1834 & 0.88 & 1.41 \\
\hline AcetyrCoA synthetase & к01895 & 1.25 & 1.25 \\
\hline Fructose-1,6-bisphosphatase I & к03841 & 0.84 & 0.79 \\
\hline Aldehyde dehydrogenase family 7 member A1 & К14085 & 0.68 & 1.03 \\
\hline Phosphoglucomutase / phosphopentomutase & K15779 & 0.97 & 1.35 \\
\hline
\end{tabular}

spliceosome, oxidative phosphorylation, glycolysis/gluconeogenesis, biosynthesis of amino acids, endocytosis, and RNA transport (Fig. 2). These results suggest that the expression of proteins involved in various metabolic pathways in piglet jejunal differentiated enterocytes changes during suckling. This present study focused on proteins related to nutrient metabolism, including glycolysis, fatty acid metabolism, amino acid metabolism, and citrate cycle.

\section{Glycolysis}

A total of 22 differentially-expressed proteins involved in glycolysis were identified in piglet jejunal upper villus enterocytes during suckling (Table 1). The expression of L-lactate dehydrogenase, pyruvate dehydrogenase E1 component subunit alpha, hexokinase, 6-phosphofructokinase 1, pyruvate kinase, fructose-bisphosphate aldolase (class I), and acetyl-CoA synthetase was upregulated in jejunal upper villus enterocytes of $14 \mathrm{~d}$ old piglets compared with that in $7 \mathrm{~d}$ old piglets (Table 1). However, the expression of S-(hydroxymethyl) glutathione dehydrogenase/Alcohol dehydrogenase, aldehyde dehydrogenase $\left(\mathrm{NAD}^{+}\right)$, glyceraldehyde 3-phosphate dehydrogenase, pyruvate dehydrogenase E1 component subunit beta, phosphoenolpyruvate carboxykinase (GTP), aldose 1-epimerase, and aldehyde dehydrogenase family 7 member A1 was down-regulated in jejunal upper villus enterocytes of $14 \mathrm{~d}$ old piglets compared with that in $7 \mathrm{~d}$ old piglets (Table 1). The expression of most of the proteins related to glycolysis was up-regulated in jejunal upper villus enterocytes of $21 \mathrm{~d}$ old piglets compared with that in $7 \mathrm{~d}$ old piglets (Table 1).
Table 2. Abundance changes of proteins involved in fatty acid metabolism injejunal upper villus enterocytes of suckling piglets

\begin{tabular}{lccc}
\hline \multirow{2}{*}{ Proteins } & \multirow{2}{*}{ KEGG } & \multicolumn{2}{c}{ Fold change } \\
& Orthology & D14 vs & D21 vs \\
& D7 & D7 \\
\hline 3-hydroxyacyl-CoA dehydrogenase & K00022 & 0.83 & 2.32 \\
Acyl-CoA oxidase & K00232 & 0.99 & 1.37 \\
Acyl-CoA dehydrogenase & K00249 & 0.68 & 0.96 \\
Long-chain-acyl-CoA dehydrogenase & K00255 & 0.75 & 0.72 \\
Acetyl-CoA C-acetyltransferase & K00626 & 0.53 & 1.00 \\
Fatty acid synthase, animal type & K00665 & 0.95 & 1.50 \\
Long-chain acyl-CoA synthetase & K01897 & 0.77 & 0.75 \\
Acetyl-CoA acyltransferase & K07509 & 0.74 & 1.18 \\
Enoyl-CoA hydratase & K07511 & 0.58 & 0.95 \\
Acetyl-CoA acyltransferase 1 & K07513 & 0.92 & 1.30 \\
Enoyl-CoA hydratase / 3-hydroxyacyl-CoA Dehydrogenase / 3,2-trans- & K07514 & 1.00 & 1.29 \\
enoyl-CoA isomerase & & & \\
Enoyl-CoA hydratase / long-chain 3-hydroxyacyl-CoA dehydrogenase & K07515 & 1.45 & 1.02 \\
Peroxisomal trans-2-enoyl-CoA reductase & K07753 & 1.25 & 1.08 \\
Carnitine O-palmitoyltransferase 1 & K08765 & 0.45 & 0.98 \\
Very long chain acyl-CoA dehydrogenase & K094779 & 0.79 & 0.74 \\
\hline
\end{tabular}

Table 3. Abundance changes of proteins involved in amino acids metabolism in jejunal upper villus enterocytes of suckling piglets

\begin{tabular}{|c|c|c|c|}
\hline \multirow[b]{2}{*}{ Proteins } & \multirow[b]{2}{*}{$\begin{array}{c}\text { KEGG } \\
\text { Orthology }\end{array}$} & \multicolumn{2}{|c|}{ Fold change } \\
\hline & & $\begin{array}{c}\text { D14 vs } \\
\text { D7 }\end{array}$ & $\begin{array}{l}\text { D21 vs } \\
\text { D7 }\end{array}$ \\
\hline $\begin{array}{l}\text { Enoyl-CoA hydratase / 3-hydroxyacyl-CoA Dehydrogenase / 3,2-trans- } \\
\text { enoyl-CoA isomerase }\end{array}$ & К07514 & 1.00 & 1.29 \\
\hline 2-oxoglutarate dehydrogenase E1 component & K00164 & 0.77 & 1.29 \\
\hline Aldehyde dehydrogenase family 9 member A1 & K00149 & 0.94 & 1.28 \\
\hline D-amino-acid oxidase & К00273 & 0.69 & 1.27 \\
\hline Carbamoyl-phosphate synthase (ammonia) & К01948 & 0.82 & 1.26 \\
\hline Methylmalonyl-CoA/ethylmalonyl-CoA epimerase & К05606 & 1.30 & 1.24 \\
\hline Alanine transaminase & К00814 & 1.88 & 1.18 \\
\hline Acetyl-CoA acyltransferase & K07509 & 0.74 & 1.18 \\
\hline 3-oxoacid CoA-transferase & К01027 & 0.68 & 1.14 \\
\hline Glucosamine--fructose-6-phosphate aminotransferase (isomerizing) & ко0820 & 1.12 & 1.13 \\
\hline Aldehyde dehydrogenase family 7 member A1 & K14085 & 0.68 & 1.03 \\
\hline Enoyl-CoA hydratase / long-chain 3-hydroxyacyl-CoA dehydrogenase & К07515 & 1.45 & 1.02 \\
\hline Acetyl-CoA C-acetyltransferase & К00626 & 0.53 & 1.00 \\
\hline Argininosuccinate synthase & К01940 & 0.27 & 0.99 \\
\hline Acyl-CoA dehydrogenase & К00249 & 0.68 & 0.96 \\
\hline Enoyl-CoA hydratase & K07511 & 0.58 & 0.95 \\
\hline Aldehyde dehydrogenase (NAD+) & К00128 & 0.58 & 0.93 \\
\hline Glutamate dehydrogenase $(\mathrm{NAD}(\mathrm{P})+)$ & К00261 & 0.67 & 0.91 \\
\hline Cytosolic nonspecific dipeptidase & К08660 & 0.64 & 0.85 \\
\hline Ornithine--oxo-acid transaminase & к00819 & 0.56 & 0.84 \\
\hline $\begin{array}{l}\text { Kynurenine---oxoglutarate transaminase / cysteine-S-conjugate beta- } \\
\text { lyase / glutamine---phenylpyruvate transaminase }\end{array}$ & К00816 & 0.76 & 0.79 \\
\hline Catalase & К03781 & 0.85 & 0.77 \\
\hline Omega-amidase & K13566 & 0.74 & 0.76 \\
\hline Diamine oxidase & K11182 & 0.67 & 0.76 \\
\hline Propionyl-CoA carboxylase beta chain & К01966 & 0.87 & 0.76 \\
\hline Argininosuccinate lyase & К01755 & 0.53 & 0.73 \\
\hline Hydroxymethylglutaryl-CoA lyase & К01640 & 0.64 & 0.72 \\
\hline Isovaleryl-CoA dehydrogenase & К00253 & 0.83 & 0.59 \\
\hline Succinate-semialdehyde dehydrogenase & ко0139 & 0.62 & 0.58 \\
\hline Aspartate aminotransferase, cytoplasmic & K14454 & 1.42 & 0.57 \\
\hline Cytosol aminopeptidase & K11142 & 0.39 & 0.44 \\
\hline 3-hydroxyanthranilate 3,4-dioxygenase & К00452 & 0.55 & 0.44 \\
\hline Glycine amidinotransferase & К00613 & 0.47 & 0.40 \\
\hline $\begin{array}{l}\text { Alanine-glyoxylate transaminase / (R)-3-amino-2-methylpropionate- } \\
\text { pyruvate transaminase }\end{array}$ & К00827 & 0.32 & 0.36 \\
\hline
\end{tabular}




\section{Fatty acid metabolism}

Fifteen proteins involved in fatty acid metabolism were differentially expressed in piglet jejunal upper-villus enterocytes during suckling (Table 2). Most of the proteins involved in fatty acid metabolism were down-regulated in jejunal upper villus enterocytes of 14 $\mathrm{d}$ old piglets compared with that in $7 \mathrm{~d}$ old piglets (Table 2). The expression of 3-hydroxyacyl-CoA dehydrogenase, acylCoA oxidase, Fatty acid synthase (animal type), acetyl-CoA acyltransferase 1 , and enoyl-CoA hydratase/3-hydroxyacyl-CoA dehydrogenase/3, 2-trans-enoyl-CoA isomerase was up-regulated in jejunal upper villus enterocytes of $21 \mathrm{~d}$ old piglets compared with that in $7 \mathrm{~d}$ old piglets, while the expression of long-chain-acylCoA dehydrogenase, long-chain acyl-CoA synthetase, and very long chain acyl-CoA dehydrogenase was down-regulated in jejunal upper villus enterocytes of $21 \mathrm{~d}$ old piglets compared with that in $7 \mathrm{~d}$ old piglets (Table 2).

\section{Amino acid metabolism}

The down-regulation of 34 differentially-expressed proteins involved in amino acid metabolism were identified in suckling piglet jejunal upper villus enterocytes (Table 3). Most of the proteins involved in amino acid metabolism (e.g. alanine, aspartate and glutamate metabolism, valine, leucine and isoleucine degradation, arginine and proline metabolism, and tryptophan metabolism) were downregulated in jejunal upper villus enterocytes of $14 \mathrm{~d}$ old piglets compared with that in $7 \mathrm{~d}$ old piglets (Table 3; Table 4-7). More enzymes involved in alanine, aspartate and glutamate metabolism, valine, leucine and isoleucine degradation, arginine and proline metabolism, and tryptophan metabolism expressed up-regulation than down-regulation in 21-day piglet jejunal upper villus enterocytes than in 7-day piglets (Table 4-7).

\section{Citrate cycle}

Seventeen proteins involved in citrate cycle differentially expressed in piglet jejunal upper villus enterocytes during suckling (Table 8). The majority of these differentiallyexpressed proteins involved in citrate cycle down-regulated in 14-day jejunal upper villus piglet enterocytes than in 7-day piglets (Table 8). Most of the differentially-expressed proteins involved in citrate cycle up-regulated in 21-day jejunal upper villus piglet enterocytes than in 7-day piglets (Table 8).
Table 4. Abundance changes of proteins involved in alanine, aspartate and glutamate metabolism injejunal upper villus enterocytes of suckling piglets

\begin{tabular}{|c|c|c|c|}
\hline \multirow[b]{2}{*}{ Proteins } & \multirow{2}{*}{$\begin{array}{c}\text { KEGG } \\
\text { Orthology }\end{array}$} & \multicolumn{2}{|c|}{ Fold change } \\
\hline & & $\begin{array}{c}\text { D14 vs } \\
\text { D7 }\end{array}$ & $\begin{array}{c}\text { D21 vs } \\
\text { D7 }\end{array}$ \\
\hline Succinate-semialdehyde dehydrogenase & K00139 & 0.62 & 0.58 \\
\hline Glutamate dehydrogenase $\left(\mathrm{NAD}(\mathrm{P})^{+}\right)$ & К00261 & 0.67 & 0.91 \\
\hline Alanine transaminase & К00814 & 1.88 & 1.18 \\
\hline $\begin{array}{l}\text { Alanine-glyoxylate transaminase / (R)-3-amino-2- } \\
\text { methylpropionate-pyruvate transaminase }\end{array}$ & К00827 & 0.32 & 0.36 \\
\hline Argininosuccinate lyase & К01755 & 0.53 & 0.73 \\
\hline Adenylosuccinate synthase & К01939 & 0.93 & 1.36 \\
\hline Argininosuccinate synthase & К01940 & 0.27 & 0.99 \\
\hline Carbamoyl-phosphate synthase (ammonia) & К01948 & 0.82 & 1.26 \\
\hline Asparagine synthase (glutamine-hydrolysing) & К01953 & 0.69 & 1.67 \\
\hline $\begin{array}{l}\text { 4-amin obutyrate aminotransferase / (S)-3-amino-2- } \\
\text { methylpropionate transaminase }\end{array}$ & K13524 & 0.94 & 1.46 \\
\hline Omega-amidase & K13566 & 0.74 & 0.76 \\
\hline Aspartate aminotransferase, cytoplasmic & K14454 & 1.42 & 0.57 \\
\hline
\end{tabular}

Table 5. Abundance changes of proteins involved in valine, leucine and isoleucine degradation injejunal upper villus enterocytes of suckling piglets

\begin{tabular}{lccc}
\hline Proteins & \multirow{2}{*}{ KEGG } & \multicolumn{2}{c}{ Fold change } \\
& Orthology & $\begin{array}{c}\text { D14 vs } \\
\text { D7 }\end{array}$ & $\begin{array}{c}\text { D21 vs } \\
\text { D7 }\end{array}$ \\
\hline 3-hydroxyisobutyrate dehydrogenase & K00020 & 0.71 & 0.49 \\
3-hydroxyacyl-CoA dehydrogenase & K00022 & 0.83 & 2.32 \\
Aldehyde dehydrogenase (NAD+) & K00128 & 0.58 & 0.93 \\
Malonate-semialdehyde dehydrogenase (acetylating) / & K00140 & 1.18 & 2.96 \\
methylmalonate-semialdehyde dehydrogenase & & & \\
Aldehyde dehydrogenase family 9 member A1 & K00149 & 0.94 & 1.28 \\
Acyl-CoA dehydrogenase & K00249 & 0.68 & 0.96 \\
Isovaleryl-CoA dehydrogenase & K00253 & 0.83 & 0.59 \\
Acetyl-CoA C-acetyltransferase & K00626 & 0.53 & 1.00 \\
3-oxoacid CoA-transferase & K01027 & 0.68 & 1.14 \\
Hydroxymethylglutaryl-CoA lyase & K01640 & 0.64 & 0.72 \\
Propionyl-CoA carboxylase beta chain & K01966 & 0.87 & 0.76 \\
Methylmalonyl-CoA/ethylmalonyl-CoA epimerase & K05606 & 1.30 & 1.24 \\
Acetyl-CoA acyltransferase & K07509 & 0.74 & 1.18 \\
Enoyl-CoA hydratase & K07511 & 0.58 & 0.95 \\
Acetyl-CoA acyltransferase 1 & K07513 & 0.92 & 1.30 \\
Enoyl-CoA hydratase / 3-hydroxyacyl-CoA dehydrogenase / 3,2-trans- & K07514 & 1.00 & 1.29 \\
enoyl-CoA isomerase & & & \\
Enoyl-CoA hydratase / long-chain 3-hydroxyacyl-COA dehydrogenase & K07515 & 1.45 & 1.02 \\
4-aminobutyrate aminotransferase / (S)-3-amino-2-methylpropionate & K13524 & 0.94 & 1.46 \\
transaminase & & & \\
Aldehyde dehydrogenase family 7 member A1 & K14085 & 0.68 & 1.03 \\
\hline & & &
\end{tabular}




\section{Discussion}

Visceral tissues, especially intestinal mucosa, have high nutrient metabolism rates [7]. Our results suggest that various biological processes in piglet DE changed during suckling. Differentially-expressed proteins were highly enriched in metabolic processes. This may be due to high intestinal enterocyte nutrient metabolism rates. Metabolic process changes in sucking pig intestinal differentiated enterocytes may be partly result from changes in the type of intestinal enterocytes resulting from vacuolated fetal-typeenterocytes replacement by non-vacuolatedadulttype in suckling piglet small intestines [6]. More research is needed to study the mechanism that affects metabolic processes of differentiated enterocytes in suckling piglets.

We further analyzed the specific metabolic pathways using KEGG pathway enrichment analysis. The results showed that the differentially-expressed proteins were primarily involved in energy metabolism (e.g., carbon metabolism, oxidative phosphorylation,glycolysis/ gluconeogenesis) and amino acids metabolism. Hansson et al. showed that biological processes related to carbohydrate metabolism, tricarboxylic acid pathway, fatty acid metabolism, amino acid metabolism, and electron transport changed in mice intestinal epithelial cells during suckling [10]. Although the intestinal epithelial cells (whole intestinal epithelial cells vs. DE) and experimental animals (mouse vs. pig) used in prior studies differ from the present experiment, the changed metabolic processes are similar. These results indicate that suckling animals may have similar metabolic changes in intestinal epithelial cells.

Intestinal mucosa nutrient metabolism is very complex. Nutrient sources for intestinal mucosa are provided by both arterial and luminal substrates [19]. The intestinal mucosa is the first tissue with the capability to use luminal nutrients. Nutrient metabolism in intestinal enterocytes can be altered by nutrients composition in the diet $[20,21]$. Nutrients metabolism in villus enterocytes differs from that in crypt
Table 6. Abundance changes of proteins involved in arginine and proline metabolism injejunal upper villus enterocytes of suckling piglets

\begin{tabular}{lccc}
\hline \multirow{2}{*}{ Proteins } & \multirow{2}{*}{ KEGG Orthology } & \multicolumn{2}{c}{ Fold change } \\
& & D14 vs D7 & D21 vs D7 \\
\hline Aldehyde dehydrogenase (NAD+) & K00128 & 0.58 & 0.93 \\
Aldehyde dehydrogenase family 9 member A1 & K00149 & 0.94 & 1.28 \\
Glutamate dehydrogenase (NAD(P)+) & K00261 & 0.67 & 0.91 \\
D-amino-acid oxidase & K00273 & 0.69 & 1.27 \\
Monoamine oxidase & K00274 & 0.71 & 1.52 \\
Glycine amidinotransferase & K00613 & 0.47 & 0.40 \\
Spermine synthase & K00802 & 0.90 & 1.38 \\
Ornithine--oxo-acid transaminase & K00819 & 0.56 & 0.84 \\
Creatine kinase & K00933 & 0.98 & 1.67 \\
Argininosuccinate lyase & K01755 & 0.53 & 0.73 \\
Argininosuccinate synthase & K01940 & 0.27 & 0.99 \\
Carbamoyl-phosphate synthase (ammonia) & K01948 & 0.82 & 1.26 \\
Cytosolic nonspecific dipeptidase & K08660 & 0.64 & 0.85 \\
Cytosol aminopeptidase & K11142 & 0.39 & 0.44 \\
Diamine oxidase & K11182 & 0.67 & 0.76 \\
Delta-1-pyrroline-5-carboxylate synthetase & K12657 & 0.85 & 1.31 \\
Aldehyde dehydrogenase family 7 member A1 & K14085 & 0.68 & 1.03 \\
Aspartate aminotransferase, cytoplasmic & K14454 & 1.42 & 0.57 \\
Argininosuccinate lyase & K01755 & 0.53 & 0.73 \\
Argininosuccinate synthase & K01940 & 0.27 & 0.99 \\
Carbamoyl-phosphate synthase (ammonia) & K01948 & 0.82 & 1.26 \\
\hline
\end{tabular}

Table 7. Abundance changes of proteins involved in tryptophan metabolism injejunal upper villus enterocytes of suckling piglets

\begin{tabular}{lccc}
\hline Proteins & KEGG & \multicolumn{2}{c}{ Fold change } \\
& Orthology & $\begin{array}{c}\text { D14 vs } \\
\text { D7 }\end{array}$ & $\begin{array}{c}\text { D21 vs } \\
\text { D7 }\end{array}$ \\
\hline 3-hydroxyacyl-CoA dehydrogenase & K00022 & 0.83 & 2.32 \\
Aldehyde dehydrogenase (NAD+) & K00128 & 0.58 & 0.93 \\
Aldehyde dehydrogenase family 9 member A1 & K00149 & 0.94 & 1.28 \\
2-oxoglutarate dehydrogenase E1 component & K00164 & 0.77 & 1.29 \\
monoamine oxidase & K00274 & 0.71 & 1.52 \\
3-hydroxyanthranilate 3,4-dioxygenase & K00452 & 0.55 & 0.44 \\
Acetyl-CoA C-acetyltransferase & K00626 & 0.53 & 1.00 \\
Kynurenine---oxoglutarate transaminase / cysteine-S-conjugate beta- & K00816 & 0.76 & 0.79 \\
lyase / glutamine---phenylpyruvate transaminase & & & \\
Catalase & K03781 & 0.85 & 0.77 \\
Enoyl-CoA hydratase & K07511 & 0.58 & 0.95 \\
Enoyl-CoA hydratase / 3-hydroxyacyl-CoA dehydrogenase / 3,2-trans- & K07514 & 1.00 & 1.29 \\
enoyl-CoA isomerase & & & \\
Enoyl-CoA hydratase / long-chain 3-hydroxyacyl-CoA dehydrogenase & K07515 & 1.45 & 1.02 \\
Diamine oxidase & K11182 & 0.67 & 0.76 \\
Aldehyde dehydrogenase family 7 member A1 & K14085 & 0.68 & 1.03 \\
\hline
\end{tabular}

Table 8. Abundance changes of proteins involved in citrate cycle injejunal upper villus enterocytes of suckling piglets

\begin{tabular}{lccc}
\hline \multirow{2}{*}{ Proteins } & \multirow{2}{*}{ KEGG } & \multicolumn{2}{c}{ Fold change } \\
& Orthology & $\begin{array}{c}\text { D14 vs } \\
\text { D7 }\end{array}$ & $\begin{array}{c}\text { D21 vs } \\
\text { D7 }\end{array}$ \\
\hline Malate dehydrogenase & K00025 & 1.78 & 2.66 \\
Malate dehydrogenase & K00026 & 0.84 & 1.31 \\
Isocitrate dehydrogenase (NAD+) & K00030 & 0.80 & 1.80 \\
Isocitrate dehydrogenase & K00031 & 0.72 & 1.36 \\
Pyruvate dehydrogenase E1 component subunit alpha & K00161 & 1.68 & 2.25 \\
Pyruvate dehydrogenase E1 component subunit beta & K00162 & 0.57 & 1.26 \\
2-oxoglutarate dehydrogenase E1 component & K00164 & 0.77 & 1.29 \\
Succinate dehydrogenase (ubiquinone) flavoprotein subunit & K00234 & 1.22 & 0.95 \\
Succinate dehydrogenase (ubiquinone) iron-sulfur subunit & K00235 & 1.09 & 1.55 \\
2-oxoglutarate dehydrogenase E2 component (dihydrolipoamide & K00658 & 1.60 & 1.40 \\
succinyltransferase) & & & \\
Phosphoenolpyruvate carboxykinase (GTP) & K01596 & 0.70 & 0.77 \\
citrate synthase & K01647 & 0.35 & 0.53 \\
Fumarate hydratase, class II & K01679 & 0.56 & 0.81 \\
Aconitate hydratase & K01681 & 0.50 & 0.75 \\
Succinyl-CoA synthetase alpha subunit & K01899 & 0.71 & 0.68 \\
Succinyl-CoA synthetase beta subunit & K01900 & 0.57 & 1.00 \\
Pyruvate carboxylase & K01958 & 1.54 & 1.42 \\
\hline
\end{tabular}


enterocytes. Glucose and fatty acids metabolism increases in villus enterocytes compared to cryptenterocytes $[22,23]$. In addition to glucose and fatty acids, amino acids, particularly glutamate and glutamine, are important energy sources for intestinal enterocytes [24, 25]. Stoll et al. showed that enteral glucose, arterial glucose, enteral glutamate, and arterial glutamine contributed $15 \%, 29 \%, 36 \%$, and $19 \%$ of the total $\mathrm{CO}_{2}$ production in piglet PDV, respectively [25]. The present experimental results show that the expression of most of the proteins involved in fatty acid metabolism and alanine, aspartate and glutamate metabolism decreased in 14-day piglet DE compared to 7-day piglets. Less than one-third of the proteins related to glycolysis decreased in 14-day piglet differentiated enterocytes compared to 7 -day piglets. The majority of all proteins related to glycolysis up-regulated in intestinal differentiated enterocytes in of 21-day piglets compared to 7-day piglets. Only a few proteins involved in fatty acid metabolism and alanine, aspartate and glutamate metabolism upregulated in intestinal differentiated enterocytes of 21-day piglets compared to 7-day piglets.

These results indicate that energy metabolism in intestinal differentiated piglet enterocytes changes during suckling. The changes in glucose, fatty acids, and alanine, aspartate and glutamate metabolism were different between early (7-14 d) and late (14-21 d) suckling piglets. Glucose, fatty acids and glutamate metabolism have different patterns in piglet intestinal DE during suckling. The nutrients for villus enterocytes, especially for upper villus enterocytes, are thought to be provided mainly by luminal substrates. Thus metabolism inupper villus enterocyte metabolism in suckling piglets can be affected by sow milk composition [26, 27]. Various experiments suggest that the contents of lactose, fatty acids, and glutamine and glutamate were changed in the sow milk [28-30]. Different sow milk may be a confounding factor in the protein expression differences found in this study. Gastrointestinal development is also regulated by genetic influences. Age-related changes in enzymes and nutrient transporters are genetically programmed and are little affected by diet [2]. This study cannot address whether these nutrient metabolism changes during suckling relate to maturity, sow milk, or genetics.

Besides the amino acids, including glutamate, glutamine, aspartate, and asparagine, that play key roles in generating energy, piglet intestines also consume many other amino acids [27]. Stoll et al. showed that $61 \%, 35 \%, 35 \%$, and $32 \%$ of the dietary Thr, Lys, Phe, and Leu, respectively, were consumed by PVD tissues during the first-pass metabolism. Only $12 \%, 18 \%, 18 \%$, and $12 \%$ of the total first-pass metabolism of Thr, Lys, Phe, and Leu were recovered in mucosal protein [31]. They concluded that about one-third of all dietary essential amino acids consumed by the intestine during the first-pass metabolism, and that more amino acids were catabolized by the mucosal cells compared to amino acids which were incorporated into mucosal protein [31].

The results of the present study showed that the metabolism of various of amino acids change in suckling piglet intestinal differentiated enterocytes. In addition, the metabolism of most of the amino acids decreased from day 7 to day 14, while only part of the detected proteins down-regulated in intestinal differentiated enterocytes of 21-day piglets compared to 7-day piglets. Consistent with energy metabolism, amino acids metabolism changes in suckling pig intestinal differentiated enterocytes may also depend on luminal nutrients as protein amounts in sow milk increased during suckling $[28,30]$.

The intestinal first-pass metabolism of dietary nutrients is independent of dietary nutrients intake [27]. It has been reported that dietary protein intake has little effect on the first-pass metabolism of essential dietary amino acids in the intestine, but may suppress arterial amino acid utilization [20, 27]. Therefore, understanding intestinal nutrients metabolism is very important for designing milk replacements for early-weaned piglets. For example, the gastrointestine may consume most of the dietary amino acids when piglets were given low-protein diets. This would restrict muscle and other organ grown and piglet overall growth [32]. The growth and development of the gastrointestinal system may be inhibited if piglets are provided with very low nutrient-content diets as luminal nutrients are essential to the gastrointestinal system. Dietary nutrients play key roles in affecting intestinal metabolism [19]. Maintaining intestinal function is critical because any decrease in enteral 
nutrients results in reduced growth and intestinal mucosa dysfunction even when piglets are provided an equal amount of nutrients parenterally [20]. The present experiment suggests that nutrient metabolism changes in suckling piglet intestinal differentiated enterocytes of piglets provides useful information for designing piglet feed formula and regulating piglet intestinal growth and development. Intestinal adaptation, which occurs following the loss of a major portion of the small intestine, has important implications to patient potential [33]. The adaptive process may be different for intestinal crypt and villus tips because the crypt and villus epithelial cells have different functions and nutrient sources [26,33]. There results of this study may also provide useful information for improving neonatal intestinal adaption.

In conclusion, the present study shows that various cellular processes in piglet intestinal differentiated enterocytes change during suckling. The metabolism of glucose, fatty acids, and alanine, aspartate and glutamate change with variously. The changes in glucose, fatty acids, and alanine, aspartate and glutamate metabolism also differ between early (7-14 day) and late (14-21 day) sucklings. These results reveal metabolic energy changes in intestinal differentiated enterocytes and may provide useful information for designing piglet feed formulas and regulating piglet intestinal growth and development.

\section{Abbreviations}

DE(differentiatedenterocytes); BSA(bovineserumalumin); PMSF (phenylmethylsulfonyl fluoride); DTT (dithiothreitol); PCNA (proliferating cell nuclear antigen); IDA (information dependant acquisition); MGF (mascot generic format); FDR (false discovery rate); KEGG (Kyoto encyclopedia of genes and genomes); GO (gene ontology); PDV (portal-drained viscera).

\section{Acknowledgements}

The authors would like to thank Dr. Chengbo Yang for superb technical assistance. The present study was jointly supported by National Natural Science Foundation of China (31330075, 31301988, 31402089), Key Programs of Frontier Scientific Research of the Chinese Academy of Sciences (QYZDY-SSW-SMC008), Natural Science Foundation of Hunan Province (2017JJ1020), Young Elite Scientists Sponsorship Program by CAST (YESS20160086).

\section{Disclosure Statement}

The authors have declared that no conflict of interests exists.

\section{References}

1 Barszcz M, Skomial J: The development of the small intestine of piglets - chosen aspects. J Anim Feed Sci 2011;20:3-15.

2 Yang H, Xiong X, Yin Y: Development and renewal of intestinal villi in pigs; in Blachier F, Wu G, Yin Y (eds): Nutritional and physiological functions of amino acids in pigs. Springere-Vienna, Vienna, 2013, pp 29-47.

- Z Zabielski R, Godlewski MM, Guilloteau P: Control of Development of Gastrointestinal System in Neonates. J Physiol Pharmacol 2008;59:35-54.

4 Xu RJ, Wang F, Zhang SH: Postnatal adaptation of the gastrointestinal tract in neonatal pigs: a possible role of milk-borne growth factors. Livest Prod Sci 2000;66:95-107. 


\section{Cellular Physiology Cell Physiol Biochem 2018;48:2103-2113 \begin{tabular}{l|l|l} 
and Biochemistry Published online: August 9, 2018 & $\begin{array}{l}\text { C) } 2018 \text { The Author(s). Published by S. Karger AG, Basel } \\
\text { www.karger.com/cpb }\end{array}$ \\
\hline
\end{tabular}}

Wang et al.: Nutrients Metabolism in Enterocytes of Piglet

5 Skrzypek T, Valverde Piedra JL, Skrzypek H, Wolinski J, Kazimierczak W, Szymanczyk S, Pawlowska M, Zabielski R: Light and scanning electron microscopy evaluation of the postnatal small intestinal mucosa development in pigs. J Physiol Pharmacol 2005;56 Suppl 3:71-87.

-6 Skrzypek T, Valverde Piedra JL, Skrzypek H, Kazimierczak W, Biernat M, Zabielski R: Gradual disappearance of vacuolated enterocytes in the small intestine of neonatal piglets. J Physiol Pharmacol 2007;58 Suppl 3:8795.

-7 Van Der Schoor SRd, Reeds PJ, Stoll B, Henry JF, Rosenberger JR, Burrin DG, Van Goudoever JB: The high metabolic cost of a functional gut. Gastroenterology 2002;123:1931-1940.

-8 McNurlan MA, Garlick PJ: Contribution of rat liver and gastrointestinal tract to whole-body protein synthesis in the rat. Biochem J 1980;186:381-383.

-9 Burrin DG, Ferrell CL, Britton RA, Bauer M: Level of nutrition and visceral organ size and metabolic activity in sheep. Br J Nutr 1990;64:439-448.

10 Hansson J, Panchaud A, Favre L, Bosco N, Mansourian R, Benyacoub J, Blum S, Jensen ON, Kussmann M: Time-resolved quantitative proteome analysis of in vivo intestinal development. Mol Cell Proteomics 2011;10:M110 005231.

11 Guilloteau P, Zabielski R, Hammon HM, Metges CC: Nutritional programming of gastrointestinal tract development. Is the pig a good model for man? Nutr Res Rev 2010;23:4-22.

12 Yang H, Xiong X, Wang X, Yin Y: Mammalian Target of Rapamycin Signaling Pathway Changes with Intestinal Epithelial Cells Renewal Along Crypt-Villus Axis. Cell Physiol Biochem 2016;39:751-759.

$\checkmark 13$ Yang H, Wang X, Xiong X, Yin Y: Energy metabolism in intestinal epithelial cells during maturation along the crypt-villus axis. Sci Rep 2016;6:31917.

$\checkmark 14$ Fan MZ, Stoll B, Jiang R, Burrin DG: Enterocyte digestive enzyme activity along the crypt-villus and longitudinal axes in the neonatal pig small intestine. J Anim Sci 2001;79:371-381.

15 Xiong X, Yang H, Tan B, Yang C, Wu M, Liu G, Kim SW, Li T, Li L, Wang J, Wu G, Yin Y: Differential expression of proteins involved in energy production along the crypt-villus axis in early-weaning pig small intestine. Am J Physiol Gastrointest Liver Physiol 2015;309:G229-237.

16 Yang H, Xiong X, Wang X, Tan B, Li T, Yin Y: Effects of Weaning on Intestinal Upper Villus Epithelial Cells of Piglets. PLoS One 2016;11:e0150216.

17 Conesa A, Gotz S, Garcia-Gomez JM, Terol J, Talon M, Robles M: Blast2GO: a universal tool for annotation, visualization and analysis in functional genomics research. Bioinformatics 2005;21:3674-3676.

18 Ye J, Fang L, Zheng H, Zhang Y, Chen J, Zhang Z, Wang J, Li S, Li R, Bolund L, Wang J: WEGO: a web tool for plotting GO annotations. Nucleic Acids Res 2006;34:W293-297.

19 Burrin DG, Stoll B, Jiang R, Chang X, Hartmann B, Holst JJ, Greeley GH, Jr., Reeds PJ: Minimal enteral nutrient requirements for intestinal growth in neonatal piglets: how much is enough? Am J Clin Nutr 2000;71:16031610.

20 van Goudoever JB, Stoll B, Henry JF, Burrin DG, Reeds PJ: Adaptive regulation of intestinal lysine metabolism. Proc Natl Acad Sci U S A 2000;97:11620-11625.

-21 van der Schoor SR, van Goudoever JB, Stoll B, Henry JF, Rosenberger JR, Burrin DG, Reeds PJ: The pattern of intestinal substrate oxidation is altered by protein restriction in pigs. Gastroenterology 2001;121:11671175.

22 Chang J, Chance MR, Nicholas C, Ahmed N, Guilmeau S, Flandez M, Wang DH, Byun DS, Nasser S, Albanese JM, Comer GA, Heerdt BG, Wilson AJ, Augenlicht LH, Mariadason JM: Proteomic changes during intestinal cell maturation in vivo. J Proteomics 2008;71:530-546.

23 Yang H, Xiong X, Yin Y: Metabolomic analysis of intestinal epithelial cell maturation along the crypt-villus axis. Rsc Advances 2016;6:27566-27574.

24 Wu G: Intestinal mucosal amino acid catabolism. J Nutr 1998;128:1249-1252.

25 Stoll B, Burrin DG, Henry J, Yu H, Jahoor F, Reeds PJ: Substrate oxidation by the portal drained viscera of fed piglets. Am J Physiol 1999;277:E168-175.

26 Alpers DH: Protein synthesis in intestinal mucosa: the effect of route of administration of precursor amino acids. J Clin Invest 1972;51:167-173.

27 Reeds PJ, Burrin DG: The gut and amino acid homeostasis. Nutrition 2000;16:666-668. 
28 Klaver J, van Kempen GJM, de Lange PGB, Verstegen MWA, Boer H: Milk Composition and Daily Yield of Different Milk Components as Affected by Sow Condition and Lactation/Feeding Regimen. J Anim Sci 1981;52:1091-1097.

29 Noblet J, Etienne M: Effect of energy level in lactating sows on yield and composition of milk and nutrient balance of piglets. J Anim Sci 1986;63:1888-1896.

30 Wu G, Knabe DA: Free and protein-bound amino acids in sow's colostrum and milk. J Nutr 1994;124:415424.

-31 Stoll B, Henry J, Reeds PJ, Yu H, Jahoor F, Burrin DG: Catabolism dominates the first-pass intestinal metabolism of dietary essential amino acids in milk protein-fed piglets. J Nutr 1998;128:606-614.

32 Ebner S, Schoknecht P, Reeds P, Burrin D: Growth and metabolism of gastrointestinal and skeletal muscle tissues in protein-malnourished neonatal pigs. Am J Physiol 1994;266:R1736-1743.

33 Drozdowski L, Thomson AB: Intestinal mucosal adaptation. World J Gastroenterol 2006;12:4614-4627. 